\title{
Artificial color tuning of firefly luminescence: Theoretical mutation by tuning electrostatic interactions between protein and luciferin
}

\section{AUTHOR(S):}

Nakatani, Naoki; Hasegawa, Jun-ya; Nakatsuji, Hiroshi

\section{CITATION:}

Nakatani, Naoki ... [et al]. Artificial color tuning of firefly luminescence: Theoretical mutation by tuning electrostatic interactions between protein and luciferin. Chemical Physics Letters 2009, 469(1-3): 191-194

\section{ISSUE DATE:}

2009-02-03

URL:

http://hdl.handle.net/2433/93439

\section{RIGHT:}

Copyright (C) 2008 Elsevier B.V.; This is not the published version. Please cite only the published version.; この論文は出版社版でありません。引 用の際には出版社版をご確認ご利用ください。 


\title{
Artificial Color Tuning of Firefly Luminescence: Theoretical Mutation by Tuning Electrostatic Interactions between Protein and Luciferin
}

\author{
Naoki Nakatani, ${ }^{a}$ Jun-ya Hasegawa, ${ }^{a, b}$ Hiroshi Nakatsuji ${ }^{a, b, *}$ \\ ${ }^{a}$ Department of Synthetic Chemistry and Biological Chemistry, Graduate School of Engineering, Kyoto \\ University, Kyoto-Daigaku-Katsura, Nishikyo-ku, Kyoto 615-8510, Japan. \\ ${ }^{\mathrm{b}}$ Quantum Chemistry Research Instritute (QCRI), JST CREST, Kyodai Katsura Venture Plaza, Goryou \\ Oohara 1-36, Nishikyo-Ku, Kyoto 615-8245, Japan \\ * Corresponding author. E-mail: h.nakatsuji@qcri.or.jp
}

\begin{abstract}
Electrostatic interactions between firefly oxyluciferin and the surrounding proteins were analyzed, and the amino acids important for controlling emission energy were identified. We propose Arg223Ala, Glu344Ala, and Asp422Ala mutations in firefly oxyluciferase of Photinus pyralis, which artificially change the luminescence color by tuning the electrostatic effect from the luciferase proteins. In the theoretical mutation simulation, the emission energy of the triple mutant was estimated to be $2.05 \mathrm{eV}$ (602 nm, reddish-orange), which is $0.18 \mathrm{eV}$ lower than that of the wild type $(2.23 \mathrm{eV}, 557 \mathrm{~nm}$, yellow-green). For calculating the emission energies, we used the symmetry-adapted cluster-configuration interaction (SACCI) method.
\end{abstract}




\section{Introduction}

Firefly luciferase (Luc) is an oxygenaze enzyme which catalyzes the light-emitting reaction. Luc oxygenates firefly luciferin and produces oxyluciferin in the electronically excited state (see Figure 1a) [1-5]. During the decay process to the ground state, keto-oxyluciferin $\left(\mathrm{oxyLH}_{2}\right)$ shows yellow-green emission $[1,2]$. The quantum yield of the luminescence is known to be very high [6]. Firefly Luc has successfully been applied to various fields of bio-imaging [2] to assay ATP generation [7], gene expression [8], and biosensors for environmental pollutants [8]. It is desirable to develop Luc showing different emission colors [2]. In particular, red-light emitting Luc variants are useful for the applications to mammalian cells which absorb shorter wavelength $[2,9]$.

Recently, a red emitting Ser284Thr mutant $(615 \mathrm{~nm}, 2.02 \mathrm{eV})$ and a green emitting Val241Ile/Gly246Ala/Phe250Ser triple mutant $(549 \mathrm{~nm}, 2.26 \mathrm{eV})$ of Photinus pyralis (Ppy) have been developed and showed favorable spectroscopic and kinetic properties [10]. A synergistic effect was observed in a mutagenesis study [11], indicating that the active-site local structure and the steric effect were related to the spectral tuning [11]. Regarding Luciola cruciata, two red emitting mutants, Ile288Val (613 nm, $2.02 \mathrm{eV})$ and Ile288Ala $(613 \mathrm{~nm}, 2.02 \mathrm{eV})$ were also produced [12]. According to the X-ray structural observations, the spectral shift correlates with the size of the side chain in the 288-th amino acid [12]. These successful examples are thus based on the steric constraint which controls the structural relaxation of the excited $\mathrm{oxyLH}_{2}$ before the emission (Figure 1b).

In our previous study [13], we analyzed the emission-color tuning mechanism of Ppy Luc, why red light in chemiluminescence and yellow-green in Luc. The $\mathrm{oxyLH}_{2}$ structural distortion induced in the Luc environment reduces the emission energy by about $0.24-0.39 \mathrm{eV}$. The $\mathrm{C}-\mathrm{C}$ dihedral angles between the two rings were ascribed as the main structural difference. This result indicated that the emission energies might be controlled by modifying the structural relaxations in the excited state of $\operatorname{oxyLH}_{2}$, which supports the steric constraint strategy [10-12].

We have also shown that the quantum and classical Coulombic interactions between $\mathrm{oxyLH}_{2}$ and $\mathrm{Luc}$ increase the emission energy by about $0.50-0.60 \mathrm{eV}$. We performed quantum-theoretical calculations and clarified the crucial role of the protein-electrostatic effects on the emission energy. Since the charge distribution of oxyLH $\mathrm{H}_{2}$ changes upon the electronic transition, the emission energy is sensitive to the protein-electrostatic 
environment as schematically shown in Figure 1c. This effect was shown to be a major factor for making the emission color to be yellow-green [13]. Therefore, it is a reasonable strategy to control the electrostatic interactions between $\mathrm{oxyLH}_{2}$ and Luc proteins. Once the interactions between $\mathrm{oxyLH}_{2}$ and amino acids are analyzed, we are able to identify several key amino acids which control the emission energy. It is therefore possible to propose new mutation experiments for the emission-spectral tuning.

For calculating emission energies, we used the Symmetry-Adapted Cluster-Configuration Interaction (SAC-CI) method [14-17]. The method has been applied to more than 150 molecules and established as an accurate electronic-structure theory for the ground and excited states [18-20]. In our recent study, we successfully reproduced the emission energies of $\mathrm{oxyLH}_{2}$ observed in chemiluminescence and bioluminescence [13]. The method also successfully applied to analyze the color-tuning mechanisms in retinal proteins [21-24] and green-fluorescent proteins $[25,26]$.

The aim of the present study is to propose mutations to control the electrostatic interactions between $\mathrm{oxyLH}_{2}$ and Luc for tuning the color of the firefly luminescence. First, the results of the decomposition analysis of the electrostatic interaction between $\operatorname{oxyLH}_{2}$ and the surrounding molecules are described. Next, on the basis of the electrostatic strategy, our new proposals of the mutations are proposed. Finally, to numerically examine our proposals, we performed the SAC-CI emission-energy calculations, and the results of the theoretical mutation simulations are described.

\section{Computational Details}

The X-ray structure (1LCI[27]) and the working model proposed in the experimental studies [28-32] were used for constructing the oxyLH $\mathrm{H}_{2}$-Luc binding complexes. Molecular dynamics calculations with AMBER94 force-field [33] were performed to find the stable structures. The force-field parameters for $\mathrm{oxyLH}_{2}$ were designed for the first excited state [13]. When all of atoms in an amino acid were located more than $7.0 \AA$ away from $\operatorname{oxyLH}_{2}$, the atomic coordinates were frozen in the molecular dynamics (MD) calculations. We took three stable structures from the trajectory. These three had different AMP conformations. There were only minor differences in the other part of the structures. Starting from these three, we raised the temperature stepwise up to $300 \mathrm{~K}$, in which total $60 \mathrm{ps}$ MD simulations were performed. Then we performed $30 \mathrm{ps}$ MD simulations at $300 \mathrm{~K}$. After the MD calculations, the molecular mechanics (MM) energy minimizations were performed. The 
MM minimized structures were used for the initial guesses for the Configuration Interaction Singles (CIS) geometry optimizations.

In the CIS calculations, oxyLH 2 , AMP, and amino acids in the first solvation layer (ARG218, HIS245, PHE247, GLY341, LEU342, SER347, ALA348, and the crystal water HOH45) were treated with the quantum mechanical $(\mathrm{QM})$ method. The rest of the protein effect was included by the classical point-charge model defined by the MM parameters. Geometry of $\mathrm{OxyLH}_{2}$ was optimized in the CIS calculations. The other atoms in the QM region were fixed to the MM optimized structures. The basis sets of 6-31g* were used for oxyLH $\mathrm{H}_{2}$, and the 6-31g set for the rest of the QM atoms.

Single-point SAC-CI calculations were performed at the optimized geometries to evaluate the fluorescence energies. $\mathrm{OxyLH}_{2}, \mathrm{His} 245, \mathrm{Arg} 218$, and the phosphate group of AMP were treated quantum mechanically, and the rest was done by a classical point-charge model. All valence orbitals were included in the active space of the SAC-CI calculations. Perturbation selection was performed to select the double excitation operators [34]. The energy thresholds of $5.0 \times 10^{-6}$ and $5.0 \times 10^{-7}$ hartree (LevelTwo) were used for the ground and excited states, respectively. For the basis sets, we used Huzinaga-Dunning double-zeta basis sets [35] augmented with the single-polarization d-function [35]. The calculated emission energy for the wild type, $2.23 \mathrm{eV}$, was in good agreement with the experimental one, $2.33 \mathrm{eV}$ [13].

In constructing the computational model, we trusted the X-ray structure. Comparing the structures available in the Protein Data Bank [27,36], the luciferase structures was not so sensitive to the inhibitor binding in the active site [36]. In our previous study [13], including the oxyluciferin in the binding site gave only small structural changes. One reason is that luciferase has open space in the binding site. If there is no inhibitor inside, the space is almost filled by water molecules.

The QM and MM calculations were performed using the Gaussian 03 [37] and TINKER programs [38-40], respectively.

\section{Result and Discussions}

3.1 Role of the protein-oxyLH $\mathrm{H}_{2}$ interactions behind the yellow-green emission of the wild type.

Since bioluminescences originate from the electronic transition, the charge distribution of the chromophore changes after the luminescence. In our previous study, the emission color tuning mechanism was 
ascribed to the increase/decrease in the charges of the carbonyl $O$ atoms [13]. However, the transition is better characterized as an intramolecular charge transfer from the benzothiazole ring to the thiazole moiety. Figure 2 shows the orbital distributions of the lowest unoccupied molecular orbital (LUMO) and the highest occupied molecular orbital (HOMO). As seen in Figure 2b, the benzothiazole ring in the HOMO is more populated than the thiazole ring. In contrast, the LUMO shown in Figure 2a distributes slightly more in the thiazole moiety. Dipole moments clearly reflect the charge-transfer character as shown in Figure $2 \mathrm{c}$. $\mathrm{OxyLH}_{2}$ is polar in the ground state $(8.45 \mathrm{D})$, but, in the excited state, the dipole moment is much smaller (3.94 D).

Due to the charge-transfer character, the emission energy of $\mathrm{oxyLH}_{2}$ is influenced by the electrostatic field generated by the Luc environment, which were the major origin of the yellow-green emission in the wild type (WT). We analyzed the protein-electrostatic effect at the amino acids resolution as follows.

$$
\begin{aligned}
\Delta E & =\sum_{M}^{\text {Amino acids }} \Delta E_{M}=\sum_{M}^{\text {Amino acids }}\left\{E_{M}^{E x}-E_{M}^{G}\right\} \\
& =\sum_{M}^{\text {Amino acids }}\left\{\sum_{B \in M} \sum_{A \in \mathrm{OxyLH}_{2}} \frac{\left(Q_{A}^{E x}-Q_{A}^{G}\right) Q_{B}}{r_{A B}}\right\}
\end{aligned}
$$

The $\Delta E$ is the difference in the interaction energy between the ground and excited states, and can be easily decomposed into the contribution from each amino acid, $\Delta E_{M}\left(=E_{M}^{E x}-E_{M}^{G}\right)$. The $E_{M}^{E x}$ and $E_{M}^{G}$ are the electrostatic interaction energies in the excited and ground states, respectively. We evaluated the interaction energy as the classical Coulombic interaction between $\mathrm{oxyLH}_{2}$ and the surrounding molecules. The atomic charges of oxyLH$H_{2}$ in the ground and the excited states, $Q_{A}^{G}$ and $Q_{A}^{E x}$, were obtained by the electrostaticpotential fitting using HF and CIS wave functions, respectively. The $Q_{B}$ for the atoms in Luc were taken from the AMBER94 force-field [33].

The result of the decomposition analysis is shown in Figure 3. The blue- and red-shift contributions in the emission energy caused by each amino acid were indicated by the positive and negative regions of the diagram, respectively. It is clearly shown that Arg218, His245, and AMP (phosphate group) give the primary contributions. Arg218 and AMP cause the blue-shifts of 0.29 and $0.21 \mathrm{eV}$, respectively, and His 245 contributes to the red-shift of $0.17 \mathrm{eV}$ [13]. In a previous experimental mutation study, fluorescence energies of Arg218Ala and Arg218Gln mutants were observed to be 2.03 (611) and $2.04 \mathrm{eV}(608 \mathrm{~nm})$, respectively, which were 
around $0.2 \mathrm{eV}$ smaller than that of the wild type [32]. These experimental results qualitatively agree to our analysis, because the positively-charged residue at the 218-th position causes the blue shift. A Ser347Ala mutation red-shift around $0.17 \mathrm{eV}$ ) [30] also supports the present theoretical result.

In this study, we focus on the secondary important contributions from the amino acids relatively distant form the binding site: blue-shift contributions from Arg223, Glu344, and Asp422, and red-shift contribution from Lys529 as shown in Figure 3. The positions and structures of these amino acids were shown in Figure 4. Since $\operatorname{Arg} 223$ is positively charged and located close to $\operatorname{Arg} 218$, this amino acid contributes the positive electrostatic potential as shown in Figure 1c. In contrast, negatively charged Glu344 and Asp422 provide the negative electrostatic potential in the same way as the AMP phosphate group.

\subsection{Artificial red-and yellow-emitting firefly luciferases: theoretical mutation simulations.}

On the basis of the analysis described above, we point out some amino acids to obtain red-emitting firefly luciferases. As shown in Figure 3, Arg223, Glu344, and Asp422 have blue-shift contributions in the emission energies of the wild type. When they are replaced by a neutral residue as alanine, the blue-shift effect should disappear, and the emission energy is expected to show red shifts. Since the distances between $\mathrm{oxyLH}_{2}$ and the target residues are around 7-12 $\AA$, we assumed the structural rearrangements around the oxy $\mathrm{LH}_{2}$ binding site causes minor effect. On the other hand, Arg218, His245, and Ser347 were excluded. Since these residues are in direct contact with oxyLH${ }_{2}$, the mutations would change the oxyLH $\mathrm{L}_{2}$ binding conformations. Once the primary binding-site residue was lost, it would be very difficult to find a reasonable binding structure between $\mathrm{oxyLH}_{2}$ and Luc in computational point of view. The experimental mutations at Arg218, His245, and Ser347 were already reported [29,30,32]. Significant spectral shifts were observed in these mutants [32], which qualitatively agree to our result of the electrostatic analysis. However, since the mutation, especially Arg218, also dropped the $\operatorname{oxyLH}_{2}$ binding capability of the luciferase, the oxygenase catalytic reactions became less efficient than that in the wild type $[29,30,32]$.

Next we performed theoretical mutation simulations. The Arg223, Glu344, and Asp422 were replaced by alanines, and the structures of the amino acids were optimized by the MM calculations. The SAC-CI calculations were performed to evaluate the fluorescence energies of the mutants. 
In Table 1, the calculated emission energies were summarized. In the Arg223Ala, Glu344Ala, and Asp422Ala mutants, the emission energies were calculated to be $2.27 \mathrm{eV}, 2.22 \mathrm{eV}$, and $2.25 \mathrm{eV}$, respectively. The estimated spectral red-shifts are $0.06 \mathrm{eV}, 0.11 \mathrm{eV}$, and $0.08 \mathrm{eV}$, respectively. The amounts of the shifts qualitatively agree with those obtained by the analysis as shown in Figure 3. Table 1 also showed the "Shifted" emission energies which were shifted by $-0.10 \mathrm{eV}$ so that the theoretical result of the wild type became equal to the experimental one. The expected emission energies of the Arg223Ala, Glu344Ala, and Asp422Ala mutants were $2.17 \mathrm{eV}(571 \mathrm{~nm}), 2.12 \mathrm{eV}(585 \mathrm{~nm})$, and $2.15 \mathrm{eV}(577 \mathrm{~nm})$, respectively. Although the amounts of the shifts are not so significant, we expect yellow emissions from these single mutants.

We further examined a triple mutant, Arg223Ala/Glu344Ala/Asp422Ala. The amount of the estimated redshift became $0.18 \mathrm{eV}$ and the expected emission energy became $2.05 \mathrm{eV}(605 \mathrm{~nm})$, which is the reddish-orange emission.

To the best of our knowledge, none of the amino acids pointed out in the present study has been tested in previous experimental mutations. Since the targeted amino acids locate relatively far from $\mathrm{oxyLH}_{2}$ binding site, the mutations would not directly change the binding site. Therefore, we expect that the mutation effects proposed based on the present and previous studies [10-12] would provide additive effects in controlling the emission energy. We strongly hope that the present theoretical predictions are tested by real mutation experiments.

\section{Conclusions}

There are several successful firefly Luc mutants showing the red and green emissions $[10,12]$. The mechanism proposed in these studies was based on the steric constraint $[11,12]$. On the other hand, we found that the protein-electrostatic effect gave the largest contribution to the blue-shift in the emission energy [13]. In this study, we proposed three amino acids for the mutation experiments, Arg223, Glu344, and Asp422 in Ppy Luc.

Theoretical mutation simulations using the SAC-CI method showed that the Arg223Ala, Glu344Ala, and Asp422Ala mutations showed spectral-red shift of $0.06,0.11$, and $0.08 \mathrm{eV}$, respectively. A triple mutant, $\operatorname{Arg} 223 \mathrm{Ala} / \mathrm{Glu} 344 \mathrm{Ala} / \mathrm{Asp} 422 \mathrm{Ala}$, showed a larger red-shift of $0.18 \mathrm{eV}$. The emission energy expected is 2.05 
$\mathrm{eV}$ (605 nm, reddish-orange). We thus wish that our proposal would add color variations in addition to the existing successful mutants $[10,12]$.

\section{Acknowledgements.}

This study was supported by a Grant-in-Aid for Creative Scientific Research, Priority area "Molecular Theory for the Real Systems", and the Global COE Program (B-09) from the Ministry of Education, Culture, Sports, Sciences, and Technology of Japan, and also by a Grant-in-Aid for Young Scientists from Computing Service Group, ACCMS and IIMC, Kyoto University. Computations were performed in the Research Center for Computational Science (Okazaki, Japan) and in Kyoto University Data Processing Center.

\section{References}

[1]E. H. White, E. Rapaport, H. H. Seliger, T. A. Hopkins, Bioorg. Chem. (1971) 92-122.

[2]V. R. Viviani, Cell. Mol. Life Sci. 59 (2002) 1833-1850.

[3]H. Isobe, Y. Takano, M. Okumura, S. Kuramitsu, K. Yamaguchi, J. Am. Chem. Soc. 127 (2005) 8667-8679.

[4]L. W. Chung, S. Hayashi, M. Lundberg, T. Nakatsu, H. Kato, K. Morokuma, J. Am. Chem. Soc. 130 (2008) 12880-12881.

[5]L. D. Vico, Y.-J. Liu, J. W. Krogh, R. Lindh, J. Phys. Chem. A 111 (2007) 8013-8019.

[6]H. H. Seliger, W. D. McElroy, Arch. Biochem. Biophys. 88 (1960) 136-141.

[7]A. K. Campbell, Chemiluminescences: Principles and Applications in Biology and Medicine., VHC, Chichester, 1988.

[8]L. H. Naylor, Biochem. Pharm. 58 (1999) 749-757.

[9]B. W. Rice, M. D. Cable, M. B. Nelson, J. Biomed. Optics 6 (2001) 432-440.

[10]B. R. Branchini, T. L. Southworth, N. F. Khattak, E. Michelini, A. Roda, Anal. Biochem. 345 (2005) 140148.

[11]B. R. Branchini, D. M. Ablamsky, J. M. Rosenman, L. Uzasci, T. L. Southworth, M. Zimmer, Biochemistry 46 (2007) 13847-13855.

[12]T. Nakatsu, S. Ichiyama, J. Hiratake, A. Saldanha, N. Kobashi, K. Sakata, H. Kato, Nature 440 (2006) 372376.

[13]N. Nakatani, J. Hasegawa, H. Nakatsuji, J. Am. Chem. Soc. 129 (2007) 8756-8765

[14]H. Nakatsuji, K. Hirao, J. Chem. Phys. 68 (1978) 2053.

[15]H. Nakatsuji, Chem. Phys. Lett. 59 (1978) 362. 
[16]H. Nakatsuji, Chem. Phys. Lett. 67 (1979) 329.

[17]H. Nakatsuji, Chem. Phys. Lett. 67 (1979) 334.

[18]H. Nakatsuji, in: J. Leszczynski (Ed.), Computational Chemistry, Revies of Current Trends. World Scientific, Singapore, 1996, p. 62-124.

[19]M. Ehara, J. Hasegawa, H. Nakatsuji, in: C. Dykstra, G. Frenking, K. Kim, G. Scuseria (Eds.), Theory and Applications of Computational Chemistry. Elsevier Science, New York, 2006.

[20]J. Hasegawa, H. Nakatsuji, in: M. Shukla, J. Leszczynsk (Eds.), Radiation Induced Molecular Phenomena in Nucleic Acid: A Comprehensive Theoretical and Experimental Analysis. Springer, 2008.

[21]K. Fujimoto, J. Hasegawa, S. Hayashi, S. Kato, H. Nakatsuji, Chem. Phys. Lett. 414 (2005) 239-242.

[22]K. Fujimoto, J. Hasegawa, S. Hayashi, H. Nakatsuji, Chem. Phys. Lett. 432 (2006) 252-256.

[23]K. Fujimoto, S. Hayashi, J. Hasegawa, H. Nakatsuji, J. Chem. Theory Comput. 3 (2007) 605-618.

[24]K. Fujimoto, J. Hasegawa, H. Nakatsuji, Chem. Phys. Letters 462 (2008) 318-320

[25]A. K. Das, J. Hasegawa, T. Miyahara, M. Ehara, H. Nakatsuji, J. Comp. Chem. 24 (2003) 1421.

[26]J. Hasegawa, K. Fujimoto, B. Swerts, T. Miyahara, H. Nakatsuji, J. Comp. Chem. 28 (2007) 2443-2452.

[27]E. Conti, N. P. Franks, P. Brick, Structure 4 (1996) 287-298.

[28]B. R. Branchini, R. A. Magyar, M. H. Murtiashaw, S. M. Anderson, M. Zimmer, Biochemistry 37 (1998) 15311-15319.

[29]B. R. Branchini, R. A. Magyar, M. H. Murtiashaw, S. M. Anderson, L. C. Helgerson, M. Zimmer, Biochemistry 38 (1999) 13223-13230.

[30]B. R. Branchini, T. L. Southworth, M. H. Murtiashaw, H. Boije, S. E. Fleet, Biochemistry 42 (2003) 10429-10436.

[31]B. R. Branchini, T. L. Southworth, M. H. Murtiashaw, R. A. Magyer, S. A. Gonzalez, M. C. Ruggiero, J. G. Stroh, Biochemistry 43 (2004) 7255-7262.

[32]B. R. Branchini, R. A. Magyar, M. H. Murtiashaw, N. C. Portier, Biochemistry 40 (2001) 2410-2418.

[33]W. D. Cornell, P. Cieplak, C. I. Bayly, I. R. Gould, K. M. Merz, D. M. Ferguson, D. C. Spellmeyer, T. Fox, J. W. Caldwell, P. A. Kollman, J. Am. Chem. Soc. 117 (1995) 5179.

[34]H. Nakatsuji, Chem. Phys. 75 (1983) 425.

[35]T. H. Dunning, P. J. Hay, in: H.F. Schaefer (Ed.), Modern Theoretical Chemistry. Plenum, New York, 1976, p. 1-28.

[36]N. P. Franks, A. Jenkins, E. Conti, W. R. Lieb, P. Brick, Biophys. J. 75 (1998) 2205-2211.

[37]M. J. Frisch, G. W. Trucks, H. B. Schlegel, G. E. Scuseria, M. A. Robb, J. R. Cheeseman, J. A. Montgomery, T. Vreven, K. N. Kudin, J. C. Burant, J. M. Millam, S. S. Iyengar, J. Tomasi, V. Barone, B. Mennucci, M. Cossi, G. Scalmani, N. Rega, G. A. Petersson, H. Nakatsuji, M. Hada, M. Ehara, K. Toyota, R. Fukuda, J. Hasegawa, M. Ishida, T. Nakajima, Y. Honda, O. Kitao, H. Nakai, M. Klene, X. Li, J. E. Knox, H. P. Hratchian, J. B. Cross, C. Adamo, J. Jaramillo, R. Gomperts, R. E. Stratmann, O. Yazyev, A. J. Austin, R. Cammi, C. Pomelli, J. W. Ochterski, P. Y. Ayala, K. Morokuma, G. A. Voth, P. Salvador, J. J. Dannenberg, V. G. Zakrzewski, S. Dapprich, A. D. Daniels, M. C. Strain, O. Farkas, D. K. Malick, A. D. 
Rabuck, K. Raghavachari, J. B. Foresman, J. V. Ortiz, Q. Cui, A. G. Baboul, S. Clifford, J. Cioslowski, B. B. Stefanov, G. Liu, A. Liashenko, P. Piskorz, I. Komaromi, R. L. Martin, D. J. Fox, T. Keith, M. A. AlLaham, C. Y. Peng, A. Nanayakkara, M. Challacombe, P. M. W. Gill, B. Johnson, W. Chen, M. W. Wong, C. Gonzalez, J. A. Pople, Gaussian03. Gaussian, Inc., Pittsburgh, 2003.

[38]J. W. Ponder, F. M. Richards, J. Comp. Chem. 8 (1987) 1016-1024.

[39]C. E. Kundrot, J. W. Ponder, F. M. Richards, J. Comp. Chem. 12 (1991) 402-409.

[40]P. Ren, J. W. Ponder, J. Phys. Chem. B 107 (2003) 5933-5947. 
Table 1

Emission energies of the mutants calculated by the SAC-CI method

\begin{tabular}{lcccc}
\hline \multirow{2}{*}{ System } & \multicolumn{3}{c}{ Theoretical } & \multirow{2}{*}{ Exptl. $^{\mathrm{b}}$} \\
\cline { 2 - 4 } & SAC-CI & $\begin{array}{c}\text { Spectral } \\
\text { shift }\end{array}$ & Shifted $^{\mathrm{a}, \mathrm{b}}$ & \\
\hline Wild type & $2.33^{\mathrm{c}}$ & - & $2.23(557)$ & $2.23(557)^{\mathrm{d}}$ \\
R223A & 2.27 & -0.06 & $2.17(571)$ & \\
E344A & 2.22 & -0.11 & $2.12(585)$ & \\
D422A & 2.25 & -0.08 & $2.15(577)$ & \\
R223A/E344A/D422A & 2.15 & -0.18 & $2.05(605)$ & \\
\hline
\end{tabular}

${ }^{a}$ Emission energies were shifted by $-0.1 \mathrm{eV}$, so that the emission energy of the wild type became equal to the experimental value. ${ }^{\mathrm{b}}$ Unit is in $\mathrm{eV}$. The numbers in parentheses are in nm unit. ${ }^{\mathrm{c}}$ Reference [13]. ${ }^{\mathrm{d}}$ Reference [10]. 


\section{Figure captions}

Fig. 1. (a)Reaction scheme of the firefly-luciferin enzyme reaction. (b)Protein-electrostatic effect on the excited state of $\operatorname{oxyLH}_{2} . \Delta \mathrm{Q}_{\mathrm{O}}(\mathrm{e}-\mathrm{g})$ is the atomic-charge difference of oxygen atoms between the excited and ground states of $\operatorname{oxyLH}_{2}\left(\Delta \mathrm{Q}_{\mathrm{O}}(\mathrm{e}-\mathrm{g})=\mathrm{Q}_{\mathrm{O}}(\mathrm{e})-\mathrm{Q}_{\mathrm{O}}(\mathrm{g})\right)$.

Fig. 2. Orbital distributions of (a)LUMO and (b)HOMO. (c)Dipole moment of $\mathrm{oxyLH}_{2}$ in the first excited and ground states.

Fig. 3. Decomposition analysis of $\Delta E\left(=E^{E x}-E^{G}\right.$, difference of the interaction energies in the ground and the excited states). $\Delta E$ was decomposed into contributions from the protein residues and AMP $\left(\Delta E_{M}\right)$. Positive and negative contributions indicate spectral blue- and red-shifts in fluorescence, respectively.

Fig. 4. Amino acids relevant to the emission color-tuning. The structure of the $\mathrm{Luc}^{-} \mathrm{oxyLH}_{2}$ complex was optimized for the first excited state of oxyLH $\mathrm{L}_{2}$. 